\title{
Strumming a Single Chemical Bond
}

\author{
Alfred J. Weymouth๑, Elisabeth Riegel, Oliver Gretz, and Franz J. Giessiblø \\ University of Regensburg, 93053 Regensburg, Germany
}

(Received 16 January 2020; revised manuscript received 2 March 2020; accepted 8 April 2020; published 11 May 2020)

\begin{abstract}
Atomic force microscopy and scanning tunneling microscopy can image the internal structure of molecules adsorbed on surfaces. One reliable method is to terminate the tip with a nonreactive adsorbate, often a single $\mathrm{CO}$ molecule, and to collect data at a close distance where Pauli repulsion plays a strong role. Lateral force microscopy, in which the tip oscillates laterally, probes similar interactions but has the unique ability to pull the $\mathrm{CO}$ over a chemical bond, load it as a torsional spring, and release it as it snaps over with each oscillation cycle. This produces measurable energy dissipation. The dissipation has a characteristic decay length in the vertical direction of $4 \mathrm{pm}$, which is 13 times smaller than the decay length in typical STM or AFM experiments.
\end{abstract}

DOI: 10.1103/PhysRevLett.124.196101

One of the most important developments in the application of frequency-modulation atomic force microscopy (FM-AFM) to the study of molecular adsorbates was the demonstration that by picking up a single $\mathrm{CO}$ molecule (a CO tip), the tip could approach the adsorbate and acquire data of the internal structure [1]. The CO tip has two key advantages: first, that the apex of the tip is known, and, second, that the adsorbate can be probed at a close distance regime [2]. FM-AFM with a CO tip has been used for many applications including identifying molecules [3], measuring the strength of a hydrogen bond [4] and determining the order of chemical bonds [5].

Lateral force microscopy is a variant of atomic force microscopy in which the tip is slid over the surface while the lateral forces are recorded [6]. Understanding the energy dissipated as the tip moves across the surface requires understanding the response of the tip to the lateral forces $[7,8]$. We perform frequency-modulation lateral force microscopy (LFM) in which the tip is driven to oscillate at a set amplitude above the surface $[9,10]$. This technique is not sensitive to forces normal to the surface, such as long-range background forces on a flat terrace, making it ideal for detecting local interactions including single spins [11]. We have previously used LFM to investigate PTCDA (3,4,9,10-perylenetetracarboxylic dianhydride) at room temperature [12]. In that work, we were sensitive to individual molecules but could not resolve intramolecular structure. In this Letter, we used LFM at low

Published by the American Physical Society under the terms of the Creative Commons Attribution 4.0 International license. Further distribution of this work must maintain attribution to the author(s) and the published article's title, journal citation, and DOI. temperature with a $\mathrm{CO}$ tip to investigate molecular adsorbates.

Experiments were performed in a CreaTec LT-STM/ AFM system operating in UHV (CreaTec Fischer \& Co. GmbH, Germany). The $\mathrm{Cu}(111)$ sample was cleaned using standard sputter and anneal cycles and a submonolayer coverage of PTCDA molecules was deposited from a home-built evaporator. Measurements were conducted at $5.8 \mathrm{~K}$. CO molecules were leaked into the chamber and we transferred one to the apex metal atom of our tip $[1,13]$, as sketched in Fig. 1(a). STM and LFM data were collected with a qPlus sensor [14] mounted such that the tip oscillates laterally $[10,15]$. All data were taken at constant height with a bias voltage of $1 \mathrm{mV}$ applied to the sample. Except where explicitly mentioned, data shown in the Letter were acquired with an amplitude $A=30 \mathrm{pm}$.

X-ray standing wave and DFT-based theoretical studies have shown that PTCDA lies roughly flat on $\mathrm{Cu}(111)$ $[16,17]$. Previous STM studies showed that PTCDA forms islands in which the molecules locally arrange in a herringbone pattern, which is better described by a large supercell [18].

Close to the surface, submolecular resolution can be observed in the STM image, as shown in Fig. 1(b). The contrast is similar to that previously reported in which a deuterium molecule at the tip was used to probe PTCDA on $\mathrm{Au}(111)$ [19].

Figure 1(c) shows LFM frequency shift $(\Delta f)$ data in which the tip oscillates in the $x$ direction. Above each molecule, the inner five rings of the molecule (the perylene core) can be seen. Assuming that the contrast in this image is primarily driven by the interaction of a flexible $\mathrm{CO}$ at the end of the metal tip [20] interacting with the molecule, we can simulate the output using a modification of the "probeparticle model" [21]. This model incorporates local interaction between the $\mathrm{O}$ atom at the terminal end of the tip 
(a)

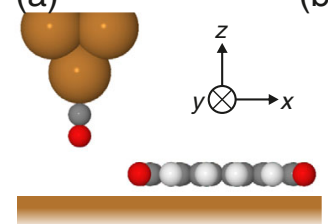

$\mathrm{Cu}(111)$

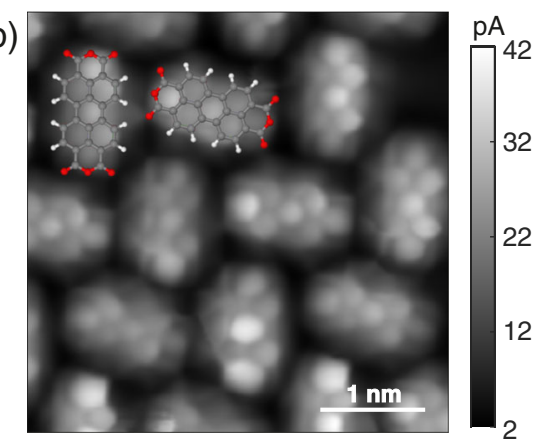

(c)

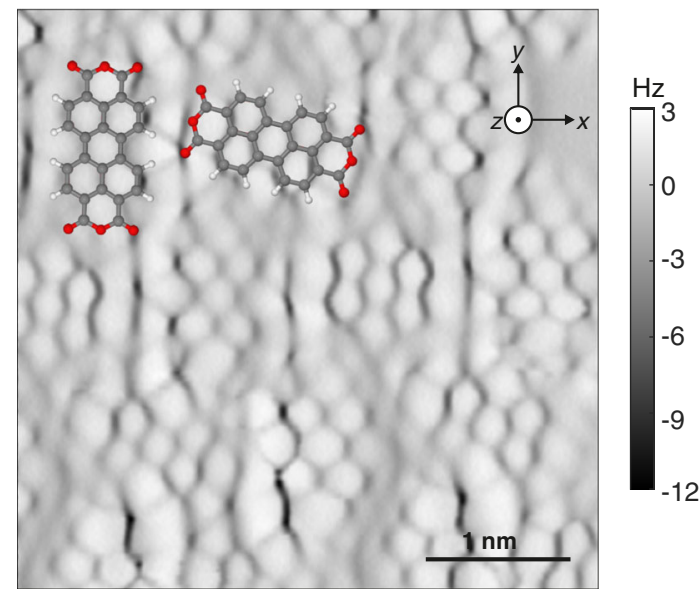

FIG. 1. PTCDA on Cu(111). (a) Sketch of the LFM setup with a $\mathrm{CO}$ adsorbed to the end of a metal tip. The tip oscillates laterally in the $x$ direction with an amplitude $A$. (b) Current image of a PTCDA island and (c) the corresponding LFM image. Molecular schematics follow the color scheme: $\mathrm{O}$ red, $\mathrm{C}$ gray, $\mathrm{H}$ white, $\mathrm{Cu}$ copper.

with surface atoms via Lennard-Jones potentials. LennardJones potentials require two parameters: the bond length and the bond energy. In the probe-particle model, an $\mathrm{O}-\mathrm{H}$ bond has length of $315 \mathrm{pm}$ and energy $2.5 \mathrm{meV}$, an O-C bond has a length of $360 \mathrm{pm}$ and an energy of $5.8 \mathrm{meV}$, and an $\mathrm{O}-\mathrm{O}$ bond has a length of $332 \mathrm{pm}$ and an energy of $9.1 \mathrm{meV}$. It has been shown that the $\mathrm{CO}$ can be modeled as a torsional spring $[22,23]$ which the probe-particle model incorporates as a classical forcefield model. We modified the probe-particle model to output the lateral force at each position of the metal apex atom. The frequency shift was calculated from the force component in the direction of the tip oscillation [24]. The output for a flat herringbone island of PTCDA is shown in Fig. S1 [25]. Although sharp lines can be seen in both the $\Delta f$ data and the model output between the molecules, they do not necessarily imply the existence of bonds (as discussed, e.g., in Ref. [26]).

From the $z$ position of Fig. 1, corresponding to $2 \mathrm{pA}$ at $1 \mathrm{mV}$ over the bare $\mathrm{Cu}(111)$ surface, we approached the tip $60 \mathrm{pm}$. As shown in Fig. 2(a), the LFM image is distorted. The dark lines over the bonds are thinner in width and show less intensity. Interestingly, there is also a strong energy loss signal shown in Fig. 2(b). In our LFM experiments, we (a)

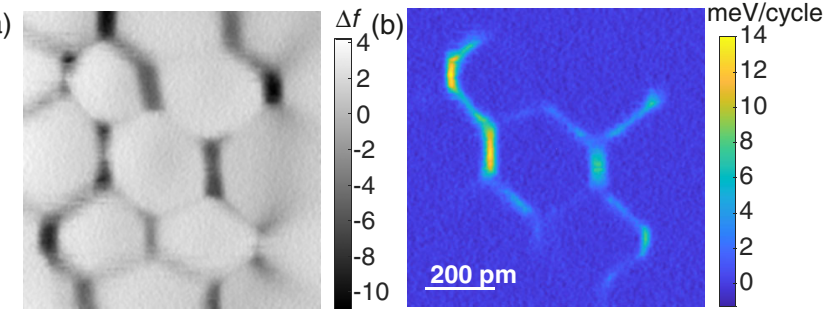

(c)
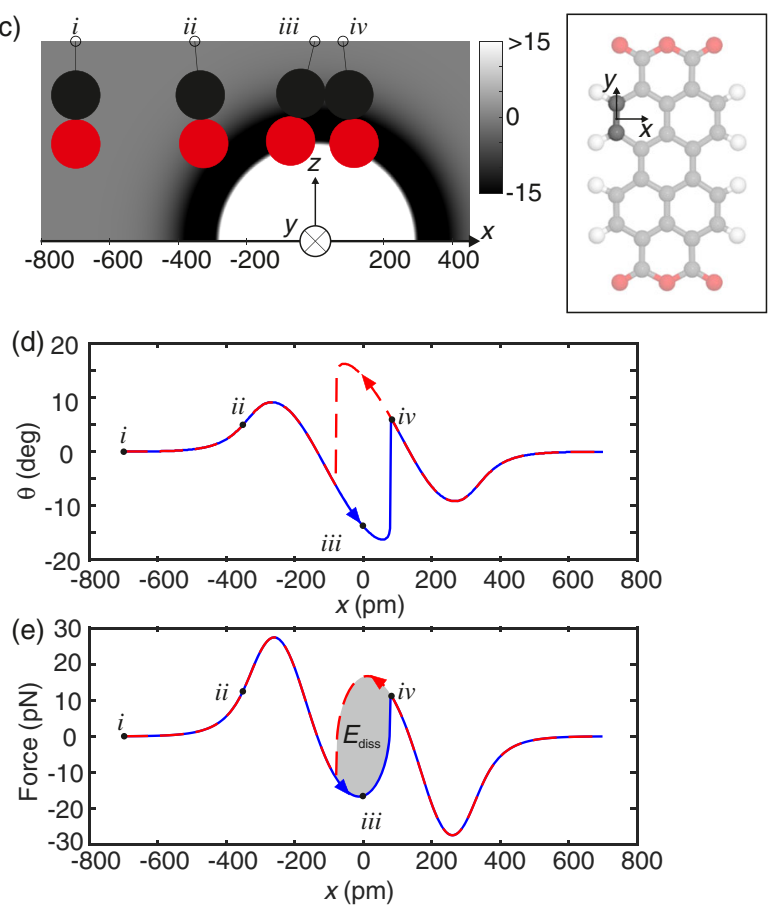

FIG. 2. Excitation in LFM data. (a) LFM image of a single PTCDA molecule and (b) corresponding excitation image. The scale bar for both (a) and (b) is shown in (b). The direction of oscillation is horizontal. (c) (Inset, right) Sketch of two atoms considered in this model. (Main) Moving the CO tip over a bond at $x=z=0 \mathrm{~m}$ causes the $\mathrm{CO}$ to tilt, but it cannot reach the universal low energy position. The background grayscale shows the potential energy (in $\mathrm{meV}$ ) of the $\mathrm{O}$ atom as acted upon by the two $\mathrm{C}$ atoms, modeled with Morse potentials. As the tip passes from left to right ( $i$ to $i i$ ), the $\mathrm{CO}$ cocks over the bond (iii) until it snaps down (iv). (d) The angle that the CO makes with the vertical as a function of the $x$ position of the metal tip apex. (e) The lateral force on the apex makes a hysteresis loop around the snap. The area of this loop is the dissipated energy, $E_{\text {diss }}$.

set the amplitude of oscillation and record the drive signal required to maintain this amplitude. If the drive signal increases, then there is energy loss as the tip oscillates, $E_{\text {diss }}$ [27]. Dissipation can be caused by different forces acting on the tip as it moves forward and backward during a single oscillation cycle [28]. This hysteretic behavior has been previously reported in systems with charges that occupy a local state for a given lifetime [29,30] or with mechanical switching of either the tip or sample [31-34].

We consider the physical mechanism of dissipation as the $\mathrm{CO}$ is driven to slide laterally over a pair of atoms. 

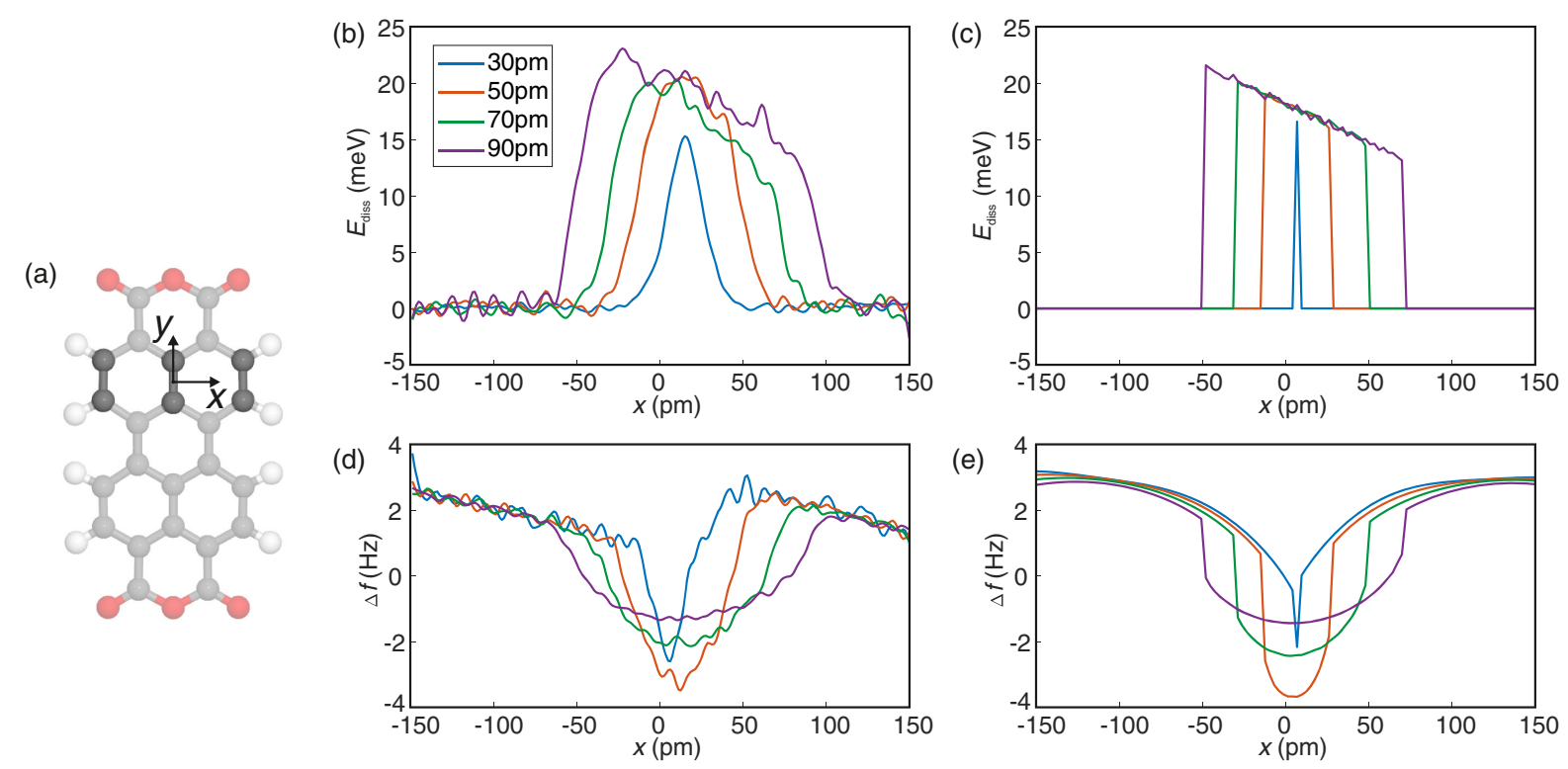

FIG. 3. Dissipation as a function of oscillation amplitude (a) The six atoms that were included in this model are shown darker than the others. (b) Experimentally measured dissipation linescans across a bond at various amplitudes can be reproduced by a snapping model shown in (c) and described in the text. (d) The frequency shift across the bonds can also be well represented by the model, shown in (e).

To do this, we reduce this problem and consider the dynamics of the simplest system: a CO tip being moved laterally over just two atoms, sketched in Fig. 2(c). The $\mathrm{O}$ atom at the tip apex interacts with two $\mathrm{C}$ atoms that are placed at $x=0 \mathrm{pm}$ and $y= \pm 70 \mathrm{pm}$, as they would be in a $\mathrm{C}_{6}$ ring. The total energy of the system is calculated by the interaction of each of the $\mathrm{C}$ atoms with the $\mathrm{O}$ atom, described by a Morse potential, and the energy stored in the $\mathrm{CO}$ as a torsional spring. Because the system has mirror symmetry in the $x, z$ plane, we limit the CO relaxation (as a torsional spring) to this plane. In this model, a Morse potential was used to describe the interatomic interaction. A similar energy dissipation can also be observed when the potential is modeled with Lennard-Jones potentials, as we demonstrate later. The parameters for the Morse potential were a bond energy of $15 \mathrm{meV}$, a bond length of $322 \mathrm{pm}$, and a decay length of $50 \mathrm{pm}$. This choice of parameters will be discussed later in the context of the data shown in Fig. 3. The $\mathrm{CO}$ was taken to be a torsional spring with a moment arm of $300 \mathrm{pm}$ [35] and a spring constant of $0.5 \mathrm{~N} / \mathrm{m}$ [26].

Far from the bond, at a position indicated by $i$ in Fig. 2(c), the lateral forces on the $\mathrm{CO}$ are negligible. The $\mathrm{CO}$ is perpendicular to the surface, as illustrated in Fig. 2(d). As it moves closer to the bond, shown at position $i i$, the CO starts to be attracted and bends towards the bond, indicated by the positive $\theta$ in Fig. 2(d). There is also a net positive lateral force on it, shown in Fig. 2(e).

Closer to the bond, there are two local energy minima for each $x$ position of the metal apex atom. This represents the $\mathrm{CO}$ being either on the left (more negative $x$ ) or right (more positive $x$ ) side of the bond. Moreover, at each position, the energy barrier between the two local energy minima can be calculated as a function of $\theta$. We assume that the $\mathrm{CO}$ is trapped in one position unless the energy barrier between the two is less than $k_{B} T$ where $T$ is the temperature in kelvin and $k_{B}$ is Boltzmann's constant. This is a major difference between the existing probe-particle model and our simulation: we do not allow the $\mathrm{CO}$ to relax via bending to the global low-energy minimum, but rather consider whether it is trapped in a local low-energy position. Determining the local low-energy position at a given position is now also a function of history (as can be seen by the existence of hysteresis in the system) and increases the computational complexity. That is why, for this investigation, we limited our modeling of the $\mathrm{CO}$ snapping to systems that have mirror symmetry in the $x$, $z$ plane.

Two low energy minima with an energy barrier between the two results in a hysteresis when moving from either left to right or, alternately, from right to left. The $\mathrm{CO}$ tilts back as the tip moves forward over the bond, loading the torsional spring, until it snaps down. It should be noted that in normal FM-AFM data collected of organic molecules, dissipation is not observed as the $\mathrm{CO}$ follows the same path forward and backward when oscillating in the $z$ direction [36]. The energy dissipation that can be measured as a flexible tip apex is slid over a single bond is therefore unique to LFM experiments.

Dissipation will only occur when the amplitude is large enough such that the motion of the tip during each cycle encompasses the entire hysteresis loop. That is, when the width of the loop is less than $2 A$. In the case of the hysteresis loop shown in Fig. 2, one would only observe dissipation with an amplitude $A \geq 80 \mathrm{pm}$. 
In order to systematically investigate this phenomenon, we collected $\Delta f$ and $E_{\text {diss }}$ data over a single bond for a variety of tip heights and amplitudes. In Fig. 3(b), $E_{\text {diss }}$ is shown for amplitudes of oscillation from 30 to $90 \mathrm{pm}$. The magnitude of the dissipated energy is approximately a boxcar function with a width that increases with amplitude. In Fig. 3(d), the corresponding $\Delta f$ linescans are shown.

Even when adjusting the interatomic potential of our two-atom model shown in Fig. 2, we were unable to reproduce the amplitude dependence. This is because the bond lengths $(322 \mathrm{pm})$ are relatively large compared to the interatomic spacing in the molecule $(140 \mathrm{pm})$. Therefore neighboring carbon atoms must also be included in our model. To retain simplicity and symmetry, we considered six carbon atoms: two at $x=-242.5 \mathrm{pm}$ and $y= \pm 70 \mathrm{pm}$; two at $x=0 \mathrm{pm}$ and $y= \pm 70 \mathrm{pm}$; and two at $x=$ $+242.5 \mathrm{pm}$ and $y= \pm 70 \mathrm{pm}$, as shown in Fig. 3(a).

In order to explain the slight tilt in the $E_{\text {diss }}$ seen in Fig. 3(b), we tilted the oscillation of the tip with respect to the $x$ axis by $1.5^{\circ}$. Although an oscillation offset angle is not typically considered in FM-AFM, small angles can have large effects in LFM. We also modelled the $\Delta f$ and $E_{\text {diss }}$ data neglecting this offset angle, shown in Fig. S2 [25].

Both the calculated $\Delta f$ and $E_{\text {diss }}$ are highly dependent upon the shape of the interatomic potential. Starting from the bond length and energy from the probe-particle model of $360 \mathrm{pm}$ and $5.8 \mathrm{meV}$ [21] and a decay length of $50 \mathrm{pm}$ [23], we adjusted them to better fit the output to our observations. We found excellent agreement with a bond energy and length of $15 \mathrm{meV}$ and $322 \mathrm{pm}$, which we also used for the two-atom simulation.

The calculated $E_{\text {diss }}$ linescans can be seen in Fig. 3(c) and the calculated $\Delta f$ in Fig. 3(e) for the experimentally explored amplitudes. No weighting factors were used and the $x$ and $y$ axes are the same for both experimental and calculated results. The widening of the $\Delta f$ and $E_{\text {diss }}$ features is well reproduced, as are their magnitudes.

We similarly acquired data over a range of $z$ distances for an amplitude of $30 \mathrm{pm}$. Approaching the tip towards the surface has a drastic effect on the magnitude of $E_{\text {diss. }}$. In Fig. 4, the maximum $E_{\text {diss }}$ over a bond is shown in black dots as a function of the tip-sample distance, where $z=$ $0 \mathrm{pm}$ corresponds to the closest approach. We observe that over a small $z$ range of less than $15 \mathrm{pm}$, the magnitude of $E_{\text {diss }}$ signal rises over the noise floor of $2 \mathrm{meV}$, increases to a maximum at $z=2 \mathrm{pm}$, and starts to decrease at even smaller distances. This strong distance dependence has to do with the width of the hysteresis loop and the height of the energy barrier as the tip approaches the bond: the increased width causes the $\mathrm{CO}$ to store more energy before it is released. This curve, and its magnitude, is well reproduced by our model, shown in the red circles. For the model, a height of $z=0 \mathrm{~m}$ corresponds to the $\mathrm{CO}$ having a moment arm of $300 \mathrm{pm}$ and the apex metal atom being $587.5 \mathrm{pm}$ above the plane of the PTCDA. Once the

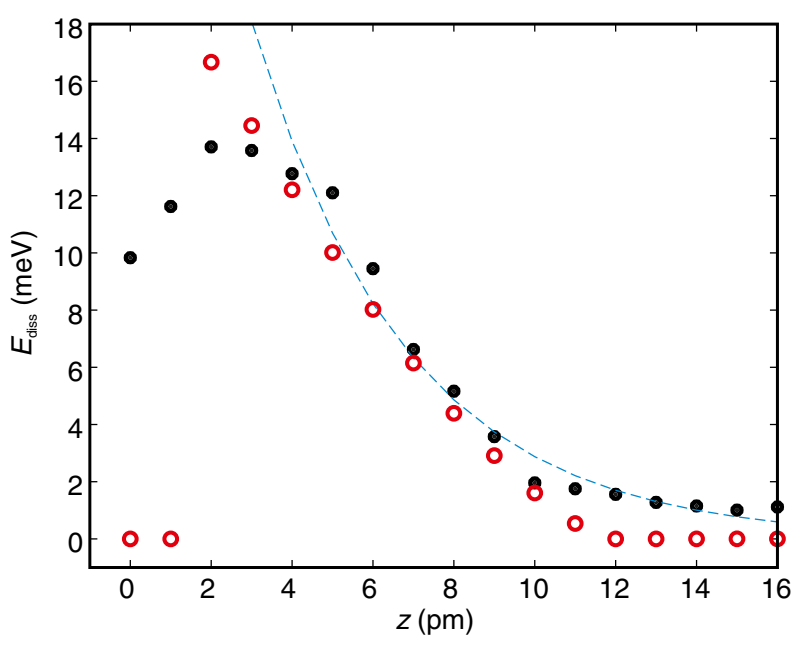

FIG. 4. The maximum energy dissipation above a bond as a function of the height of the tip. Black dots are data, red circles are the model output. The blue line is an exponential fit to the data from $z=5$ to $15 \mathrm{pm}$, to characterize the decay length.

tip is too close, the $\mathrm{CO}$ can no longer snap over it each oscillation cycle and $E_{\text {diss }}$ decreases, as seen in both the data and the model. In order to evaluate the decay length of this signal we fit the initial increase to an exponential function. This yielded a characteristic length of $4 \mathrm{pm}$, in contrast to the typical value of $54 \mathrm{pm}$ for STM [37] or AFM [38].

Line scans of the data and the model are shown in Fig. S3 [25] with the same model as for the calculations in Fig. 3. The thinning of the bonds and the decrease in the $\Delta f$ magnitude, as can be seen in Fig. 2(a), are also reproduced.

Finally, we calculated the CO dynamics over bonds using the parameters found in the widely accepted probeparticle model (many of which are taken from the AMBER force field simulation) [21]. The results are shown in Fig. S4 [25] and are very similar to those discussed above, with the excitation signal increasing in magnitude and decreasing in width as the tip approaches the surface. The agreement with our data was poorer, which was not surprising, as we did not adjust the interatomic potential. Nonetheless, both the simple six-atom model and the more complex model including the entire PTCDA molecule describe the physics behind the observed energy dissipation.

The force applied to the $\mathrm{CO}$ molecule is equal and opposite to what is applied to the bond. This means that the loading and releasing of the $\mathrm{CO}$ molecule is strumming over a single chemical bond; loading it with a lateral force and releasing it. We foresee future studies based on this work in which individual bonds of a molecular adsorbate are mechanically excited and either the decay of the resulting oscillation measured with high-speed investigations [39] or tip-enhanced Raman spectroscopy is used to identify the vibrational mode associated with energy dissipation [40]. 
The authors would like to thank A. Liebig for careful reading of the manuscript. A. J.W. and O. G. thank the Deutsche Forschungsgemeinschaft Grant No. 397771090 , "Locally mapping conductance and potential energy of a donor-acceptor system."

*jay.weymouth@ur.de

[1] L. Gross, F. Mohn, N. Moll, P. Liljeroth, and G. Meyer, The chemical structure of a molecule resolved by atomic force microscopy, Science 325, 1110 (2009).

[2] N. Moll, L. Gross, F. Mohn, A. Curioni, and G. Meyer, The mechanisms underlying the enhanced resolution of atomic force microscopy with functionalized tips, New J. Phys. 12, 125020 (2010).

[3] L. Gross, F. Mohn, N. Moll, G. Meyer, R. Ebel, W. M. Abdel-Mageed, and M. Jaspars, Organic structure determination using atomic-resolution scanning probe microscopy, Nat. Chem. 2, 821 (2010).

[4] S. Kawai, T. Nishiuchi, T. Kodama, P. Spijker, R. Pawlak, T. Meier, J. Tracey, T. Kubo, E. Meyer, and A. S. Foster, Direct quantitative measurement of the $\mathrm{C}=\mathrm{O} \ldots \mathrm{H}-\mathrm{C}$ bond by atomic force microscopy, Sci. Adv. 3, e1603258 (2017).

[5] K. Kaiser, L. M. Scriven, F. Schulz, P. Gawel, L. Gross, and H. L. Anderson, An sp-hybridized molecular carbon allotrope, cyclo[18]carbon, Science 365, 1299 (2019).

[6] C. M. Mate, G. M. McClelland, R. Erlandsson, and S. Chiang, Atomic-Scale Friction of a Tungsten Tip on a Graphite Surface, Phys. Rev. Lett. 59, 1942 (1987).

[7] L. Prandtl, Ein Gedankenmodell zur kinetischen Theorie der festen Körper, Z. Angew. Math. Mech. 8, 85 (1928).

[8] D. Tománek, W. Zhong, and H. Thomas, Calculation of an atomically modulated friction force in atomic-force microscopy, Europhys. Lett. 15, 887 (1991).

[9] O. Pfeiffer, R. Bennewitz, A. Baratoff, E. Meyer, and P. Grütter, Lateral-force measurements in dynamic force microscopy, Phys. Rev. B 65, 161403(R) (2002).

[10] F. J. Giessibl, M. Herz, and J. Mannhart, Friction traced to the single atom, Proc. Natl. Acad. Sci. U.S.A. 99, 12006 (2002).

[11] D. Rugar, R. Budakian, H. J. Mamin, and B. W. Chui, Single spin detection by magnetic resonance force microscopy, Nature (London) 430, 329 (2004).

[12] A. J. Weymouth, E. Riegel, S. Matencio, and F. J. Giessibl, Evaluating the potential energy landscape over single molecules at room temperature with lateral force microscopy, Appl. Phys. Lett. 112, 181601 (2018).

[13] L. Bartels, G. Meyer, and K.-H. Rieder, Controlled vertical manipulation of single $\mathrm{CO}$ molecules with the scanning tunneling microscope: A route to chemical contrast, Appl. Phys. Lett. 71, 213 (1997).

[14] F. J. Giessibl, High-speed force sensor for force microscopy and profilometry utilizing a quartz tuning fork, Appl. Phys. Lett. 73, 3956 (1998).

[15] A. J. Weymouth, Non-contact lateral force microscopy, J. Phys. Condens. Matter 29, 323001 (2017).

[16] A. Gerlach, S. Sellner, F. Schreiber, N. Koch, and J. Zegenhagen, Substrate-dependent bonding distances of
PTCDA: A comparative x-ray standing-wave study on $\mathrm{Cu}(111)$ and $\mathrm{Ag}$ (111), Phys. Rev. B 75, 045401 (2007).

[17] V. G. Ruiz, W. Liu, E. Zojer, M. Scheffler, and A. Tkatchenko, Density-Functional Theory with Screened van der Waals Interactions for the Modeling of Hybrid Inorganic-Organic Systems, Phys. Rev. Lett. 108, 146103 (2012).

[18] T. Wagner, A. Bannani, C. Bobisch, H. Karacuban, and R. Möller, The initial growth of PTCDA on $\mathrm{Cu}(111)$ studied by STM, J. Phys. Condens. Matter 19, 056009 (2007).

[19] C. Weiss, C. Wagner, C. Kleimann, M. Rohlfing, F. S. Tautz, and R. Temirov, Imaging Pauli Repulsion in Scanning Tunneling Microscopy, Phys. Rev. Lett. 105, 086103 (2010).

[20] L. Gross, F. Mohn, N. Moll, B. Schuler, A. Criado, E. Guitián, D. Peńa, A. Gourdon, and G. Meyer, Bond-order discrimination by atomic force microscopy, Science 337, 1326 (2012).

[21] P. Hapala, G. Kichin, C. Wagner, F. S. Tautz, R. Temirov, and P. Jelínek, Mechanism of high-resolution STM/AFM imaging with functionalized tips, Phys. Rev. B 90, 085421 (2014).

[22] M. P. Boneschanscher, S. K. Hämäläinen, P. Liljeroth, and I. Swart, Sample corrugation affects the apparent bond lengths in atomic force microscopy, ACS Nano 8, 3006 (2014).

[23] A. J. Weymouth, T. Hofmann, and F. J. Giessibl, Quantifying molecular stiffness and interaction with lateral force microscopy, Science 343, 1120 (2014).

[24] F. J. Giessibl, Forces and frequency shifts in atomic-resolution dynamic-force microscopy, Phys. Rev. B 56, 16010 (1997).

[25] See the Supplemental Material at http://link.aps.org/ supplemental/10.1103/PhysRevLett.124.196101 for additional information about the materials and methods, as well as supplemental figures 1 to 4 . Code for the strumming simulation is provided as FindAngleCC.m and CalcEdissDf.m.

[26] P. Jelinek, High resolution SPM imaging of organic molecules with functionalized tips, J. Phys. Condens. Matter 29, 343002 (2017).

[27] F. J. Giessibl, Advances in atomic force microscopy, Rev. Mod. Phys. 75, 949 (2003).

[28] H. Hölscher, B. Gotsmann, W. Allers, U. D. Schwarz, H. Fuchs, and R. Wiesendanger, Measurement of conservative and dissipative tip-sample interaction forces with a dynamic force microscope using the frequency modulation technique, Phys. Rev. B 64, 075402 (2001).

[29] M. Ondráček, P. Hapala, and P. Jelínek, Charge-state dynamics in electrostatic force spectroscopy, Nanotechnology $\mathbf{2 7}, 274005$ (2016).

[30] L. L. Patera, F. Queck, P. Scheuerer, and J. Repp, Mapping orbital changes upon electron transfer with tunnelling microscopy on insulators, Nature (London) 566, 245 (2019).

[31] S. Hembacher, F. J. Giessibl, J. Mannhart, and C. F. Quate, Local Spectroscopy and Atomic Imaging of Tunneling Current, Forces, and Dissipation on Graphite, Phys. Rev. Lett. 94, 056101 (2005).

[32] N. Oyabu, P. Pou, Y. Sugimoto, P. Jelinek, M. Abe, S. Morita, R. Pérez, and Ó. Custance, Single Atomic Contact Adhesion and Dissipation in Dynamic Force Microscopy, Phys. Rev. Lett. 96, 106101 (2006). 
[33] D. Sawada, Y. Sugimoto, M. Abe, and S. Morita, Observation of subsurface atoms of the $\mathrm{Si}(111)-(7 \times 7)$ surface by atomic force microscopy, Appl. Phys. Express 3, 116602 (2010).

[34] S. A. Ghasemi, S. Goedecker, A. Baratoff, T. Lenosky, E. Meyer, and H. J. Hug, Ubiquitous Mechanisms of Energy Dissipation in Noncontact Atomic Force Microscopy, Phys. Rev. Lett. 100, 236106 (2008).

[35] M. Gajdoš and J. Hafner, CO adsorption on $\mathrm{Cu}(111)$ and $\mathrm{Cu}(001)$ surfaces: Improving site preference in DFT calculations, Surf. Sci. 590, 117 (2005).

[36] C. Moreno, O. Stetsovych, T. K. Shimizu, and O. Custance, Imaging three-dimensional surface objects with submolecular resolution by atomic force microscopy, Nano Lett. 15, 2257 (2015).

[37] G. Binnig, H. Rohrer, C. Gerber, and E. Weibel, Tunneling through a controllable vacuum gap, Appl. Phys. Lett. 40, 178 (1982).

[38] F. J. Giessibl, Atomic resolution of the silicon $(111)-(7 \times 7)$ surface by atomic force microscopy, Science 267, 68 (1995).

[39] T. L. Cocker, D. Peller, P. Yu, J. Repp, and R. Huber, Tracking the ultrafast motion of a single molecule by femtosecond orbital imaging, Nature (London) 539, 263 (2016).

[40] J. Lee, K. T. Crampton, N. Tallarida, and V. A. Apkarian, Visualizing vibrational normal modes of a single molecule with atomically confined light, Nature (London) 568, 78 (2019). 